\title{
Philosophiques
}

\section{La pensée écoféministe : le féminisme devant le défi global de l'ère techno-scientifique}

\section{Marie-Josée Marin}

Volume 21, numéro 2, automne 1994

Les femmes et la société nouvelle

URI : https://id.erudit.org/iderudit/027279ar

DOI : https://doi.org/10.7202/027279ar

Aller au sommaire du numéro

Éditeur(s)

Société de philosophie du Québec

ISSN

0316-2923 (imprimé)

1492-1391 (numérique)

Découvrir la revue

Citer ce document

Marin, M.-J. (1994). La pensée écoféministe : le féminisme devant le défi global de l'ère techno-scientifique. Philosophiques, 21(2), 365-380.

https://doi.org/10.7202/027279ar
Résumé de l'article

La critique féministe a dénoncé très tôt le « bonheur parfait » que la techno-science est censée apporter à nos sociétés occidentales. Mais c'est la tendance écoféministe qui a poussé le plus à fond cette critique. Nous voulons mettre en évidence les principaux enjeux identifiés par ce courant, ainsi que les types de praxis qu'il veut mettre en oeuvre pour nous libérer de l'idéologie du " progrès » et nous permettre d'accéder à une société plus humaine. 


\title{
La pensée écoféministe : le féminisme devant le défi global de l'ère techno-scientifique
}

\author{
par \\ Marie-Josée Morin
}

\begin{abstract}
RÉSUME : La critique féministe a dénoncé très tôt le «bonheur parfait » que la techno-science est censée apporter à nos sociétés occidentales. Mais c'est la tendance écoféministe qui a poussé le plus à fond cette critique. Nous voulons mettre en évidence les principaux enjeux identifiés par ce courant, ainsi que les types de praxis qu'il veut mettre en œuvre pour nous libérer de l'idéologie du « progrès 》 et nous permettre d'accéder à une société plus humaine.
\end{abstract}

ABSTRACT : The feminist critique very early on denounced the «bonheur parfait» that techno-science is expected to yield in our Western societies. This line of criticism was insisted upon by ecofeminists. We would like to underscore the main issues identified by eco-feminists, together with the type of praxis it implies in order to liberate us from a progress-based ideology and to allow us acces to a more human society.

Depuis des décennies déjà, les sociétés occidentales prospères sont entrées dans une ère techno-scientifique sans précédent. Ce processus foudroyant de technologisation nécessite, au fur et à mesure qu'il prend forme dans nos praxis quotidiennes, l'établissement de nouvelles structures intellectuelles pour gérer ses implications et ses possibilités. Ainsi, depuis quelques années, les théoricien-nes de l'humain se sont vus confronté-e-s à la nécessité de la création de nouveaux modèles éthiques, épistémologiques, sociaux, politiques et métaphysiques afin, en quelque sorte, de « rattraper » les créations du « Progrès ».

Selon l'opinion de certaines gens, les découvertes et les inventions techno-scientifiques n'obéissent plus qu'à leurs propres règles, à la logique interne générée par le simple fait de leur existence : comme si la réalisation avait précédé l'intention... ou plutôt comme si la réalisation venait maintenant gouverner l'intention. 
Pour d'autres, au contraire, l'humanité pénètre dans un modèle technosocial véritablement créatif, où elle n'est plus simplement soumise au règne des nécessités naturelles, mais où il lui est enfin possible de choisir ses manières d'être-au-monde, et ainsi d'être vraiment libre... Faut-il penser que les uns ont raison et que les autres ont tort ? Je dirais plutôt que nous nous trouvons devant des positions qui reflètent des considérations incontournables, points de vue divergeants qui racontent la chronique d'un monde en réelle mutation...

Face à ces bouleversements, les féministes ne sont certes pas demeurées inactives : en fait, elles furent parmi les premières à mettre en question le supposé « bonheur parfait » unissant les sociétés occidentales et les projets et productions techno-scientifiques. Nombre d'entre elles cherchèrent à dégager les préjugés androcentristes, ethnocentristes, et de classe qui obscurcissaient les jugements en cette matière, et à mettre au jour les implications négatives et les « effets secondaires » pervers d'une grande partie de ces découvertes.

C'est dans ce cadre critique et réflexif que s'inscrit la position écoféministe. Pour mieux comprendre quels types de critiques elle formule envers les sociétés actuelles, tout autant que le modèle socio-politique sousentendu par cette pensée somme toute récente, je présenterai d'abord le plan d'ensemble dans lequel s'inscrivent ces remarques critiques. L'effort que je vous propose est compréhensif, évocateur, plutôt que simplement descriptif, et il n'a aucune prétention à l'exhaustivité. Il s'agira d'abord d'examiner des pistes de réflexion, des notions, des idéaux et des craintes, celles qui, en majeure partie, constituent les matériaux de base pour l'élaboration de cette perspective « éco-logique » sur notre existence en tant qu'animal pensant, sexué, historique, esthète et créatif, mais aussi destructeur, et jusqu'à l'autodestruction.

Nous verrons ainsi succinctement les principaux enjeux que ce courant s'attache à relever, ainsi que les types de praxis qu'il entend mettre en œuvre afin de constituer une alternative réelle à ce que l'on pourrait appeler «l'idéologie du progrès ».

L'agenda de l'écoféminisme, tendance difficile à cerner car ce mouvement se refuse bien souvent à une définition de lui-même qui soit fixe et limitative, regroupe en fait beaucoup des principales interrogations et préoccupations disséminées dans les divers mouvements féministes : ce qui pourrait donc en faire une entité idéologique en soi, est sans doute plutôt à rechercher dans sa MANiÈre d'aborder les problématiques. Il est d'ailleurs fort révélateur que le terme lui-même (écoféminisme) soit très peu employé dans la documentation, les participantes à cette pensée refusant souvent la restriction étroite d'une dénomination précise : en fait, c'est toute cette manière étroite de juger et de classer les choses, individus, comportements ou idées, qu'elles tentent primordialement de remettre en question. 


\section{Regard panoramique sur la situation abordée par l'écoféminisme}

Il est indéniable qu'avec l'irruption dans nos vies des techno-sciences modernes ${ }^{\mathrm{I}}$, la tâche de l'éthique et du jugement politique s'est radicalement modifiée. Les considérations sur notre place dans la Nature en tant qu'humains, nos droits et nos devoirs pour reprendre l'expression désignée, sont passées du champ des diktats de la religion à celui du choix démocratique et de l'engagement social individuel. En quelques décennies, nos sociétés se sont retrouvées sans les balises (normes, idéaux et critères) qui les avaient jusqu'alors guidées et, en grande partie, érigées. Aujourd'hui, ces questions sur les liens qui unissent l'humain et le monde sont devenues non plus simplement l'objet des discours philosophiques et dogmatiques, mais bien au contraire, le sujet d'un enjeu crucial dont, dans le meilleur des cas, la responsabilité des engagements (face au futur) devrait être transférée des institutions politico-idéologiques à tous les individus humains.

Cette nouvelle dynamique (éthico-sociale) pose aussi la question de la définition des sphères publiques, puisque avec les technologies modernes, les responsabilités personnelles des humains envers la nature doivent aussi se placer au centre du politique, engageant par là une redéfinition des frontières de la polis et la création de nouveaux ordres de priorités.

Hans Jonas affirme qu'il s'agit là d'une « forme de responsabilité métaphysique, au-delà de l'intérêt personnel, qui nous incombe à cause de l'immensité de notre puissance face à ce voile ténu de vie (cette mince et fragile biosphère); ce qui signifie, une responsabilité qui est la nôtre depuis que

I. Techno-sciences modernes : les nouvelles bio-technologies (NTRH; manipulations génétiques; techniques de maintien de la vie biologique; techniques de testages neurologiques, cellulaires, bio-chimiques; clònage cellulaire, bactériologiques, voir même d'organes ou de spécimens animaux - humains ? etc.), les développements informatiques et optiques (lasers) sans lesquels ces recherches sont impensables, les développements des télécommunications et des modes de transport, le développement de l'énergie nucléaire.

A noter que ni moi, ni l'immense majorité des théoriciennes écoféministes, ne maintiendrons raisonnablement que ces développements techno-scientifiques ne doivent pas être accomplis : en fait, la question ne se pose pas let nous perdrions un temps précieux à nous la poser aujourd'hui, puisque nous devons prendre des mesures d'urgence. Il est absolument dommageable pour les sociétés de devoir réagir avant d'être en mesure de connaître plus exactement le sens et la portée de leurs actes, mais c'est là la situation dans laquelle nous nous trouvons, encore une fois). Dans quelques décennies, alors qu'il sera évidemment trop tard pour faire marche arrière, peut-être l'humanité s'octroiera-t-elle un temps de repos afin de voir ce qui a mal tourné. Mais comme bien d'autres, les écoféministes chuchottent que tout cela n'aura pas si mal tourné, si nous pouvons encore nous poser des questions sur notre passé dans un siècle ou deux... 
l'homme est devenu dangereux non seulement pour lui-même, mais pour toute la biosphère. »(traduction libre) $)^{2}$.

Les questionnements qu'imposent les techno-sciences doivent donc se saisir sous l'angle essentiel de la responsabilité, et la critique écoféministe les considère en priorisant ces aspects :

I. les coûts humains du progrès;

2. la nécessité de limiter le développement techno-scientifique (ou du moins de le freiner pendant un moment);

3. les déficiences et insuffisances inhérentes à la prise de décision dans un ordre technologique;

4. ainsi que, évidemment, l'urgence de la conservation et du respect des différentes expressions de la nature, inévitablement en conjonction avec nos propres souhaits de survie sur notre planète ${ }^{3}$.

D'un autre côté, depuis quelques décennies, de nouveaux discours écologistes ont plus spécifiquement travaillé au renforcement de la relation humains-nature et à une féroce dénonciation du processus menant à la dévalorisation et à la destruction de la vie sur la planète.

Simultanément à l'émergence de ces discours qui mettaient de mieux en mieux en relief les modes de l'oppression ancestrale de la nature par l'homme et qui cherchaient à fonder la valeur intrinsèque de la nature non humaine, certaines féministes se sont mises à penser que le projet féministe devrait muter, pour qu'en priorité il soulève le défi réel, celui de donner un statut politique à la nature. La nature qui a toujours été partie prenante de la dialectique politicosociale, entité opprimée par la culture androcentriste au même titre que les femmes, et selon la même dynamique, avec, au fondement de ce processus d'infériorisation, les mêmes peurs et les mêmes enjeux. Ces féministes se sont donc lentement engagées dans cette voie, pour le moins révolutionnaire à l'intérieur des pratiques « traditionnelles » du féminisme, de réconciliation avec la nature et de reconnaissance d'une complicité historiquement (sinon ontologiquement) acquise. En termes clairs, il s'agissait de découvrir l'assise

2. «A kind of metaphysical responsability beyond self-interest has devolted in us with the magnitude of our powers relative to this tenuous film of life, that is, since man become dangerous not only to himself but to the whole biosphere. 》 (Jonas, 1984, p. 136).

3. En ce sens, si l'écoféminisme tend à ce que la perception qu'il engage soit plus large qu'une vision univoquement anthropocentrique, ce n'est pas non plus une pensée hystérique comme celle (écologiste) que l'on caricature volontiers en disant d'elle qu'elle est bien prête à sauver les baleines et les rhinocéros, mais aussi à se débarrasser d'un bon nombre d'individus humains... Certains penseurs tentent de répandre cette idée qu'une pensée véritablement éco-centriste ne peut être qu'anti-humaniste, et que la valorisation de la biosphère ne peut se réaliser qu'à l'encontre des intérêts humains : en quelque sorte, ils sont les tenants modernes de la scission métaphysique cartésienne, validant à leur tour l'irréconciliable fossé séparant la nature et la culture. 
théorique, politique et culturelle, et peut-être aussi métaphysique, qui puisse unir femmes et nature non humaine dans une lutte commune contre l'oppression patriarcale.

Les causes historiques de cette complicité entre les femmes et la nature non humaine sont identifiées de multiples façons dans la théorie écoféministe : en fait, il s'y trouve de multiples hypothèses, qui sont bien sûr invérifiables quant aux faits, mais qui possèdent des qualités explicatives ou démonstratives plus ou moins satisfaisantes. Ces hypothèses théoriques dont la portée factuelle est donc indéfinissable tiennent lieu de théories généalogiques, en quelque sorte mythes explicateurs des origines de l'infériorisation et de la dévalorisation, à la fois de la femme et de la nature non humaine.

La plus répandue, avec quelques variantes, stipule que les hommes lou le pouvoir androcentrique) ont identifié les femmes et la nature non humaine et ont cherché à les définir selon des normes idéologiques afin de raffermir le caractère masculin des puissances économique et politique. Simultanément, ils concouraient ainsi à un autre objectif d'un niveau plus symbolique (mais d'une portée politique tout aussi ambitieuse) en altérant l'image « sacralisée » des pouvoirs procréateurs des femmes. Il est clair que le message idéologique selon lequel les femmes sont plus près de la nature est essentiel à la réussite d'un tel projet, si les conditions préalables enracinant l'infériorité de la nature non humaine sont déjà réalisées. Les tenantes de cette hypothèse sont bien souvent celles qui partagent l'idée d'une forme de matriarcat préalable à l'ordre patriarcal, matriarcat fondé sur la puissance évocatrice et mystique de la procréation féminine, lieu de l'engendrement, de la création de la vie, source de pouvoir absolu en des temps où seul le poids démographique décidait de la survie ou de la disparition du groupe. Pour ces penseures, si la procréation féminine a d'abord été source de respect et même de puissance magique, elle ne peut bien sûr être cause de l'infériorisation des femmes : il faut pour cela qu'un facteur extérieur intervienne, et ce facteur, c'est la domestication de la nature, dévalorisée puisque soumise, dès lors, aux travaux de l'homme. C'est ultérieurement sur cette infériorisation et démythification de la nature non humaine que se basera tout le processus d'assimilation des femmes à l'imagerie «terrienne », puis, directement, à leur propre infériorisation et oppression.

D'autres penseures supposent, quant à elles, que les bases politiques de la domination (de tous les types de domination politique) se retrouvent dans les structures de la dévalorisation sexuale, et non dans une dévalorisation d'abord présente dans les relations conceptuelles et matérielles de l'homme et de la nature non humaine, ensuite transférée dans les rapports entre femmes et hommes. Pour les tenantes de cette première hypothèse, après la possibilité d'une période de mysticisme rituel autour de la maternité et de l'enfantement, bien rapidement les normes de l'animalité ont été greffées à celles-ci. La « découverte » de la paternité biologique, par ailleurs, a largement contribué à une repolarisation des caractéristiques « essentielles » de la reproduction, qui 
auraient été prestement associées au principe mâle, achevant ainsi le processus de dévalorisation du rôle «naturel » de la femme dans la société humaine. Puis, avec la sédentarisation progressive des sociétés, c'est l'imagerie de la reproductrice humaine, soumise et infériorisée, qui allait se transférer aux conceptions de la nature non humaine, terre sans valeur si elle ne donnait pas de fruits, terre docile et sans voix.

Mais au-delà de la diversité des points de vue, la question fondamentale qui se retrouve au premier plan de la réflexion écoféministe concerne la possibilité émancipatoire du rapport femme/nature. La théorie féministe libérale traditionnelle soutient (faut-il rappeler l'énoncé de Simone de Beauvoir ?) que cette relation n'offre que le champ d'une argumentation féconde pour la perpétuation de la subordination des femmes. C'est prioritairement cet énoncé que l'écoféminisme entend renverser, en procédant à une certaine unification des intérêts de ces deux entités opprimées sur lesquelles s'est érigé le patriarcat.

\section{Incapacité des principaux courants féministes à relever le défi des techno-sciences. Réponse de l'écoféminisme : n'ayons pas peur de nos relations avec la nature!}

La «formule » écoféministe avance que « les femmes ont été le sacrifice de la culture à la nature ${ }^{4}$. Selon ces théories, les « grandes » civilisations, conquérantes par définition, devaient, pour se construire, élaborer des notions qui allaient définir l'avancement de l'humanité selon le degré de son extirpation de la sphère naturelle. Elles allaient non seulement faire en sorte que les civilisations s'érigent sur la nature, mais à l'encontre d'elle: elles allaient créer une idéologie si puissante qu'elle bloquerait la route à tout retour en arrière ${ }^{5}$, et rendrait nécessaire le développement croissant de techniques de domination (et de manipulation) de la nature.

C'est là une des conclusions des thèses généalogiques de l'écoféminisme, qui affirme que l'infériorisation de la nature non humaine est indissociable de l'établissement de la politique patriarcale : ainsi la colonisation des « espaces vides », l'instrumentalisation des forces de la nature (devenues « ressources ») et la maîtrise de l'environnement seront alors considérées comme des «victoires » du genre humain (lire : de l'homme). Dans les plus extrêmes des cas (l'apogée du mécanicisme aux XVIII ${ }^{e}$ et XIX ${ }^{e}$ siècles, le techno-scientisme des années 1930 à 1950, l'industrialisation pollueuse et déshumanisante du

4. Selon les notions de pratiques sacrificielles en cours dans l'inconscient collectif et les pratiques de l'époque, fermement reprises par les principales religions institutionalisées. Termes employés par Ynestra King (1990).

5. Vers un mode de vie soumis à la nature, nomade, cueilleur-chasseur. A noter qu'il est généralement admis que les sociétés de chasseurs-cueilleurs accordaient une grande place aux divinités féminines, symbole de la fertilité, de la générosité et de la magie de la production de la nature. (Voir James 1960). 
début du siècle), certaines thèses plus ou moins formelles mais néanmoins largement influentes (et souvent socialement bien en vue) colporteront des idées selon lesquelles la survie de l'homme en tant qu'espèce (sic) doit être envisagée comme une lutte incessante contre les forces impitoyables et dévastatrices de la nature. Cette constante demeure : au travers des millénaires de l'histoire occidentale, les rapports des hommes avec la nature furent situés dans une dynamique de colonisation; l'humanité était responsable de la lourde tâche d'humaniser ce qui est sauvage, païen, infertile, « perdu ».

Ce discours, qui identifie la nature comme l'ennemie, donc comme celle qui rôde à l'extérieur des enclaves humanisées, se dédouble pour rappeler que la nature est aussi insidieusement présente à l'intérieur de la société; car nul ne peut nier que la plupart des activités humaines sont communes aux animaux : reproduction, croissance, alimentation, souffrance et vieillissement ne sont pas que des prérogatives humaines... C'est cette incapacité de contester l'animalité humaine qui devra être «transcendée » par un mensonge idéologique, lequel constituerait, selon ce point de vue écoféministe, le lieu de l'articulation des principaux dualismes qui vont fonder les idéologies les plus prospères de l'histoire de la pensée. Car ainsi, le dualisme nature-culture se transforme, à l'intérieur de la sphère humaine, en une dualité corps-esprit. Et, ce n'est plus désormais un secret pour qui que ce soit, cette dualité est plus poignante, incontournablement incarnée dans le processus reproductif, chez les femmes... Dès lors, toutes les activités spécifiques aux femmes seront représentées comme non historiques, non sociales, innées ou intuitives, bref, plus naturelles et moins civilisées.

Ce qui constitue fondamentalement et authentiquement l'humain, l'essence de la nature humaine, a été défini en termes intellectuels, sphère de la masculinité, mais aussi en termes servant à maximiser la distance posée entre les deux éléments de la dichotomie : la nature intouchée face à la culture transcendante, l'animal et l'homme parfaitement civilisé, l'esprit désincarné et le corps englué dans ses instincts. Exactement comme la masculinité est définie en termes de distance maximale et d'exclusion des caractéristiques féminines (ce qui permet de déterminer une « vertu » différente pour chaque sexe...), l'humanité, la notion d'humanité pleine et entière, a historiquement été définie en termes de négation et d'exclusion des caractéristiques identifiées comme non humaines, ces éléments appartenant à la sphère de l'animalité et de la nature. Comme le note Mary Midgley, l'homme enracine toutes les caractéristiques qu'il valorise en des capacités auxquelles l'animal ne peut en aucun cas prétendre. Ce refus de tout le pan naturel et animal de l'humanité, et la fixation d'un concept de vertu qui maximise sa distance face à la nature, a été nommé par l'auteur John Rodman « l'impératif de la différence » (《the differential imperative »). Ce qui crée l'humanité n'est pas seulement sa distance de la nature et du corps, mais aussi la capacité de transcender et de contrôler la nature par le biais de l'intellect et, de cette façon, de réaliser la liberté et la « pleine humanité ». 


\section{Esquisses des réponses et contre-argumentations féministes}

Après l'évocation de ce tableau général des bases de l'oppression selon la pensée écoféministe, les similitudes et les différences de celle-ci avec les principaux courants de pensée féministe semblent se dessiner un peu mieux. Mais pour parfaire le tableau des distinctions, des ajouts et des retraits théoriques que l'écoféminisme présente aux féminismes «traditionnels », nous allons maintenant succinctement évoquer les différentes prises de position des principaux courants de pensée féministe en ce qui concerne leurs orientations respectives sur les rapports unissant la femme et la nature non humaine. Ceci permettra de cerner un peu mieux en quoi elles sont, prétendent les écoféministes, insuffisantes pour la création d'une ligne de pensée apte à la compréhension de la globalité des enjeux posés par la redéfinition des liens de la nature et de la praxis technocentrique.

A notre époque, le traditionnel refus du féminisme libéral d'accepter le postulat de la «nature plus naturelle de la femme » a mené à une plus grande valorisation de la contribution des femmes dans un modèle culturel que l'on a résolument cherché à « asexualiser $»^{6}$. Mais on a accusé le féminisme libéral de ne chercher qu'à « masculiniser » les femmes, rejetant quelquefois très cavalièrement toute possibilité de réelle différence entre hommes et femmes, et faisant des caractéristiques mâles le modèle à partir duquel tracer les lignes de la « femme future ». Sa volonté affirmée de refuser la discussion de thèmes se rapportant aux relations des femmes et de la nature disqualifie a priori, pour les considérations qui nous intéressent, sa capacité de fournir un canevas sur lequel penser le «nouveau contrat » naturel que nous devons produire ${ }^{7}$.

De son côté, le féminisme rationaliste radical refuse aussi la connexion femme/culture. Pour les féministes de cette appartenance, la sortie de l'oppression consiste en la libération de la sphère primordiale de la nature, qu'elles conçoivent comme une forme de ghetto. Elles affirment que la clé de l'émancipation des femmes se trouve dans la dissociation des femmes et de la nature, et dans la fin du « ghetto femelle », une sphère de nécessité - non libre par définition. Dans ce sens, le féminisme libéral est similaire au féminisme rationaliste radical, car bien que la notion de « nature » constitue, dans les

6. En anglais, « de-genderification ».

7. Cette incapacité peut être exemplifiée par l'absence de prise de position sur le problème de la maternité de substitution, que les féministes libérales justifient en disant que, réglementer la location d'utérus reviendrait à poser une intervention étatique dans un domaine (le commerce) d'où il doit à tout prix être exclu. Le refus de percevoir les implications socio-politiques de fond, les implications culturelles techno-scientifiques qui prévalent, bien avant les intérêts de la protection du libre-marché, montre bien à quel point le libéralisme ne peut prendre en compte les transformations techno-scientifiques (surtout en ce qui concerne les NTRH) d'un point de vue critique. 
considérations radicalistes, une catégorie d'analyse centrale, elle n'y est jamais considérée comme potentiellement libératrice : en fait, c'est tout le contraire. Les positions les plus ambiguës vont jusqu'à avancer que la domination se basant sur les différences physiologiques féminines est à tout le moins compréhensible, sinon excusable. Mais, bien sûr, cette position poursuit du même souffle que les conditions socio-historiques s'étant modifiées, cette discrimination, si elle possédait une forme de légitimité antérieurement, ne peut plus aujourd'hui être acceptée.

Les féministes rationalistes radicales déplorent l'appropriation de l'écologie par le féminisme, et la perçoivent comme une régression destinée à renforcer le « stéréotypage » des genres. Tout ce qui peut renforcer les différences génériques ou poser n'importe quelle sorte de revendication en rapport avec de soi-disant spécificités féminines est problématique.

Pour Simone de Beauvoir (1986) par exemple, la figure de proue du féminisme radical rationaliste, la femme deviendra pleinement humaine, tout comme l'est l'homme, en le rejoignant, en se distanciant, en transcendant et en contrôlant sa « nature ». Elle oppose la transcendance et la conquête de la nature du mâle à l'immanence de la femme identifiée à et submergée par la nature : son corps. Ainsi la « pleine humanité » peut être atteinte par l'accession à la sphère supérieure de l'esprit, la domination et la transcendance de la nature et du physique; accession à la sphère de la liberté et de la malléabilité, contrastant avec le fait d'être immergée dans la nature et prise dans ce non-contrôle aveugle. Les femmes deviendront donc «pleinement humaines » en étant absorbées dans la sphère masculine de la liberté et de la transcendance, conceptualisée en termes humains, mais chauvinistes. Pour certaines, représentées par exemple par Shulamith Firestone (1972), la réelle libération de la femme passera par l'abandon des facultés reproductives, remplacées par une « cybernétique procréative » : encore une fois, l'incapacité à prendre en considération les bases politico-culturelles de ces transformations technologiques invalide spontanément toute possibilité d'établissement d'un point d'assise critique.

Le féminisme culturel radical, quant à lui, pose les racines de l'oppression des femmes dans une théorie diamétralement opposée, et propose une stratégie de lutte elle aussi opposée à celle du radicalisme rationaliste. Le féminisme culturel résout le problème non pas en oblitérant les différences entre les femmes et les hommes, mais en prenant le parti des femmes, qu'il dit aussi être celui de la Nature non humaine. Le féminisme culturel émerge du féminisme radical, mais en mettant l'emphase sur les différences plutôt que les similitudes entre les femmes et les hommes. Et, sans surprise, ce courant s'est approprié le slogan « le personnel est politique » en en renversant la polarité, en personnalisant le politique. Les féministes culturelles ont cherché à articuler, et même à créer une culture féminine séparée; elles ont constitué la majeure partie des tenantes de la réunion des intérêts des femmes avec la nature et la lutte féministe avec l'offensive écologiste. En effet, la force 
principale du féminisme radical culturel réside dans un mouvement profondément pro-femmes. Leur participation aux divers types de manifestations pacifistes ou anti-militaires, par exemple, passe difficilement inaperçue, tout comme leur activisme pro-autochtone dans les deux Amériques. En fait, s'il fallait tracer une généalogie, le féminisme radical culturel y serait sans aucun doute l'une des « mères » de l'écoféminisme, l'un des courants les plus fertiles où il s'est abreuvé : l'acceptation de différences fondamentales entre les principes mâle et femelle de l'espèce humaine, d'abord, puis la conceptualisation des forces émancipatoires au centre de la féminitude et de la procréation féminine, tracent l'essentiel de la parenté rattachant l'écoféminisme et le féminisme culturel radical. Cependant, sa difficulté à articuler les dynamiques dialectiques de l'histoire rend ce dernier incapable de réellement constituer une base d'analyse critique valable, en regard des nouvelles pratiques techno-scientifiques.

Enfin, en ce qui a trait au féminisme socialiste, longtemps prédominant en termes de production académique, la plupart des critiques écoféministes affirment qu'il n'est pas non plus équipé pour faire face aux défis lancés par les techno-sciences, ni apte à produire une analyse globale des rapports entre femme et nature non humaine. En effet, le féminisme socialiste ayant fait de la notion de «travail » sa catégorie centrale d'analyse, il est tombé dans le piège de la réduction de l'être humain à un «homo laborans ». De plus, et c'est sans doute là sa lacune la plus évidente, il se base en grande partie sur des notions de travail et de productivité qui ne prennent pas en compte le travail reproductif des femmes, ce qui a comme résultat direct de priver les femmes à la fois d'une base identitaire forte et de les placer à la merci des dogmes technocratiques en tout ce qui regarde les NTRH.

\section{Une réponse plausible : l'écoféminisme}

L'enjeu premier de l'écoféminisme, enjeu à la fois éthique, politique et inévitablement social, est de démontrer à partir de quels présupposés et pour quels motifs la déqualification des femmes, l'infériorisation de la nature et l'oppression patriarcale ont été pratiquées.

La dynamique patriarcale, unissant dans les rôles de victimes la femme et la nature, constitue certes un élément d'analyse large et flou qui autorise (et peut-être même nécessite) une multiplicité de points de vue sur les causes de cette conjonction, permettant de percevoir par là même les grandes lignes idéologiques qui pourraient expliquer d'un même souffle certaines des articulations communes à l'ensemble du projet patriarcal. Donc, la pensée écoféministe, comme d'ailleurs le féminisme culturel, vient requestionner la négation de la possibilité d'un rapport différent unissant les femmes et la nature (différent de celui qu'entretiennent les hommes et cette même (?) nature). Mais qu'est-ce que l'écoféminisme? 
Comme son nom (néologisme un peu rebutant) l'indique, l'écoféminisme constitue un point de vue théorique critique né de la rencontre des intérêts féministes et écologiques. Ceci étant dit, nous n'avons pas dit grand-chose...

La position écoféministe affirme que la nature non humaine et les femmes sont victimes d'un même phénomène d'oppression patriarcale, et que ces deux types d'oppression sont en fait l'expression d'un profond malaise conceptuel et d'une terreur, ou d'un refus, de la reproductivité féminine.

L'écoute de l'expérience féminine et celle de la nature sont donc deux bases d'une même transformation, et leur urgence témoigne de la destructivité du pouvoir patriarcal laissé à lui-même. Les préoccupations féministes et écologistes se rencontrent ici pour former une unité de pensée et d'action qui fournit la possibilité d'une transformation radicale de la future société. comme le féminisme, l'écoféminisme contient une forte composante utopique.

Celle-ci prend racine dans des postulats selon lesquels la nature non humaine possède une valeur propre, hors du champ purement utilitariste, et que, comme telle, elle doit être respectée. La vision écoféministe, tout comme l'écologisme, propose une reconsidération de nos besoins face aux ressources de la nature, mais aussi une reconsidération des besoins de la nature devant ce qu'il nous est possible, et que nous avons la responsabilité de lui fournir. Elle ne présente pas comme tel un refus de la technologie, mais renie complètement son mode de production et d'utilisation ${ }^{8}$.

Les femmes, par la procréation, s'inscrivent plus facilement dans le champ de la nature non humaine, ce qui pourrait constituer l'esquisse d'une validation « naturelle » de cette union; et, historiquement, cette complicité fut par ailleurs politiquement réitérée par leur commune position d'objet utilisé par le patriarcat pour « asseoir » ses pouvoirs économico-culturels. Mais, dans un premier temps, les femmes n'ont pas totalement rompu les attaches avec la « naturalité originelle » en demeurant reproductrices (voilà ce que les NTRH mettent en question), et se sentent, selon certaines écoféministes ${ }^{9}$, plus facilement engagées dans et responsables de l'avenir, ou du moins de penser l'avenir, car ce sont leurs enfants qu'elles y plongent. " Les femmes, affirme Ruddick (p. 186), ont tendance à comprendre, d'une manière ou à un degré que les hommes partagent peu, à la fois l'histoire et le coût de la chair humaine. »

La revendication politique la plus directement enracinée dans l'actualité historique de l'écoféminisme est celle de la redistribution des droits et responsabilités en matière de bio-technologie aux communautés concernées; car c'est une évidence que les prêtres de la techno-science (compagnies

8. Pourquoi crée-t-on de nouvelles technologies, pour quels intérêts, quels besoins, quelles nécessités ? Où, comment et pour pallier quoi exactement les met-on en branle, les exploite-t-on?

9. Comme Sara Ruddick (I99o). 
pharmaceutiques, laboratoires de recherches génétiques, etc.) sont les plus mal placés pour poser des jugements généraux sur les effets et les implications profondes de ces nouvelles pratiques. La principale critique à l'endroit des techno-sciences concerne leur volonté d'éviter le développement de pratiques auto-questionnantes, et de perpétuer ainsi une longue lignée de «sciences sans conscience », grâce à l'excuse, aujourd'hui heureusement criante, que la science concerne l'étude neutre de faits objectifs.

En fait, nos sociétés se trouvent à une croisée des chemins, poussées à s'engager dans un «progrès » dont il leur est impossible de mesurer l'ampleur exacte, pas même toutes les conséquences qui en découleront directement. Un regard historique permet de percevoir, entre des moments de guerres et de conquêtes territoriales hypertrophiés par les discours patriarcaux, de véritables « rond-points » politiques, culturels ou intellectuels (métaphysiques, religieux, philosophiques, techniques, esthétiques, scientifiques, etc.), où se sont joués des enjeux d'une importance telle qu'ils allaient largement définir les sociétés futures : que serait le visage de l'humanité, par exemple, sans la révolution copernicienne, sans la notion de dialectique historique, sans la prise en charge du développement technique par une « idéologie du progrès », sans la découverte de l'énergie nucléaire?

Or, il ne faut pas de dispositions particulières pour saisir que nous passons le seuil d'un bouleversement majeur de ce type, qui amènera des transformations des pratiques personnelles, privées, selon des normes, des techniques et des jugements qui sont et seront effectués par et pour des objectifs étatiques, èconomiques, si rien n'est profondément modifié. Cette croiseee des chemins est en fait celle des mutations de pratiques personnelles et communautaires (par exemple, le traitement de la procréation humaine, de la santé, de la nature, de la consommation individuelle), mutations qui nous engagent face à l'avenir, face à nos descendants, qui engagent la planète et aussi, la définition future de l'humanité ${ }^{\mathrm{TO}}$.

« Le féminisme est simplement la croyance en la pleine humanité de la femme et en son droit de se définir elle-même », disait Simone de Beauvoir. Mais en fait, cela ne peut pas être aussi simple. Parce que la notion d'humanité elle-même se doit d'être révisée sous l'impact de la transformation des pratiques sociales imposée par le nouvel ordre des techno-sciences. Et tout ce qui concerne les relations de l'humain avec la nature non humaine doit être intégré globalement à la reconsidération de ce que cela signifie d'être (politiquement et matériellement) humain. Ces concepts doivent être soumis à un examen et à une réévaluation, soutient la pensée écoféministe, parce que le développement de la notion d'humanité a été fondamentalement influencé, modélisé par les dichotomies corps/esprit et féminin/masculin.

Io. Voir Jonas (ig84). 
L'effort théorique à fournir dès aujourd'hui serait donc celui d'une définition de l'humain qui soit contextuelle (c'est-à-dire qui prenne en considération le fait que l'humain n'est qu'un des participants aux écosystèmes extrêmement complexes desquels il dépend), et qui intègre, pour cette raison même, la responsabilité d'un agir global, toujours éthique, au centre de son identité morale. Or cet effort requiert une reconstruction critique de l'épistémologie.

Les écoféministes tentent en effet de construire des modèles théoriques hybrides, qui ne font pas appel à des modes de conceptualisation isolant la rationalité de l'expérience vécue, ou les normes de l'émotion qui s'y rattache. Cette recherche d'une connaissance qui aspire à une plus grande assimilation des conditions de l'humain, qui refuse de s'amputer d'une part de sa réalité pour faire face aux enjeux éthiques et politiques qui l'attendent, peut, à mon avis, insuffler une dimension de recherche (au sens large du terme) nouvelle dans ce vaste mouvement de reconsidération de la responsabilité comme fondement décisionnel politique.

Mais en fait, cette tentative d'assimilation des dimensions traditionnellement scindées par la création des sphères privée et publique doit, pour être véritablement significative, participer d'un projet de restructuration épistémologique plus large, qui considère le savoir comme une part de la praxis humaine. Dans cette optique, il n'y a évidemment pas de raison légitimant la séparation de la raison en ordre théorétique et pratique, ou l'élaboration d'une antithèse entre les processus cognitifs de découverte des « réalités naturelles » (celles qui prévalent dans l'ordre de la nature non humaine) et les processus décisionnels (traditionnellement propres aux dimensions éthiques de l'agir humain). Cette scission, qui s'exprime dans les longues listes de dualismes soutenant les traditions philosophiques critiquées, apparaît effectivement valide dans un système épistémologique qui considère la connaissance comme participant d'une révélation, qu'elle soit rationaliste (Descartes), ou empiriste (Aristote). Mais dès lors que la connaissance perd son statut de révélation sur un ordre de choses fixe, et qu'elle apparait comme un processus multidimensionnel dont l'objet, tout autant que le sujet, est toujours à construire, il est clair que les procédés de validation du savoir doivent, eux aussi, se soumettre à une réévaluation fondamentale.

Il s'agirait alors, semble-t-il, de poser une alternative aux processus de conceptualisation et de transformation techno-scientifiques (qui sont ceux de nos sociétés actuelles), globalement érigés sous le signe du dualisme, afin de reconstruire une voie épistémologique valable qui ne tombe pas dans les écueils d'une pensée dont la finalité s'est avérée l'auto-destruction, sans pour autant sacrifier notre praxis historique de connaissance approfondie des causes et des conséquences. Le projet n'est donc pas vraiment celui de mettre sur pied une nouvelle intelligibilité, mais plutôt de proposer un modèle - tout aussi propre à notre processus conceptuel mais dont nous avons méconnu la valeur - d'organisation des connaissances qui ne tende pas inéluctablement à la manipulation, qui soit plus qualitatif et moins quantitatif, qui soit processuel 
plutôt qu'eschatologique, et dont la résultante conceptuelle soit plus fluide, plus contextuelle, et plus éthiquement concernée.

D'un même souffle, il faut créer un modèle épistémologique qui, à l'inverse des paradigmes traditionnels articulés autour d'une vision instrumentale de leur objet (selon laquelle la connaissance parfaite d'un objet est achevée lorsqu' on peut le soumettre à la volonté humaine, et, proportionnellement, c'est lorsque la technologie s'est appropriée la malléabilité d'un objet qu'on peut affirmer qu'il est connu), doit prioriser le regard auto-réflexif, constamment sous le signe de la responsabilité, sur l'objet de la connaissance.

Dans l'optique écoféministe, cela semble signifier la sortie d'un stade d'immaturité techno-scientifique et le passage à celui d'une conscience éthicoscientifique. Là se situe la nécessité d'une révision épistémologique, concomitante de celles des champs politique, économique et éthique, et vice-versa. Il ne s'agit pas de rejeter une façon d'établir des observations scientifiques théoriques pour la remplacer par une autre, mais de réaliser la nécessité complémentaire de ces deux approches (schématiquement : éthique et scientifique), leur parfaite concordance, alors que depuis des siècles nous nous acharnons à affirmer que de telles préoccupations ne sont pas réconciliables sinon par les règles (arbitraires) de la religion, ou encore, dans le meilleur des cas, qu'elles se rejoignent mais selon des ordres hiérarchiques constitués à partir de dogmes intellectuels traditionnels.

Or, selon l'écoféminisme, il faut cesser de penser à travers les lunettes déformantes des dualismes radicaux et toujours simplistes, et passer à un type de conceptualisation qui est certes plus complexe, requiert plus d'éléments et un processus compréhensif plus large, mais dont nous ne pouvons plus, dès maintenant, faire l'économie. Axer la perception et le jugement des formes théoriques vers des compréhensions en continuum plutôt que morcelées, enracinées dans l'histoire et le mouvement socio-politique plutôt que décontextualisées, participantes d'une cohérence culturelle plutôt qu'universelle, intégrées à une expérience significative plutôt que transcendante.

Comme nous l'avons mentionné, la pensée écoféministe ne va pas jusqu'à prôner l'abolition des formes de savoir scientifico-démonstratives, ou revendiquer la disparition de tout concept universel ou de toute norme générale : il s'agirait là, outre d'un cul-de-sac conceptuel, de la répétition du même schéma dogmatique qui a proliféré jusqu'à aujourd'hui, c'est-à-dire la dévalorisation d'un type d'intellection jumelée à une absolue consécration d'un autre, perçu de préférence de manière antagoniste. Et fait, il doit alors s'agir d'une fusion plus que d'un remplacement : fusion des intérêts, des types de processus de connaissance et de validation de jugement; changement de priorités qui pourra peut-être ultimement, aux yeux des écoféministes, mener à une «transmutation » des processus cognitifs-scientifiques.

Les alternatives à la pensée dualiste - une épistémologie intégrant la raison et l'émotion, l'intellect et les sens; une métaphysique alternative qui intègre l'esprit et le corps et qui rejette le dualisme fondamental de la position 
mécaniste en place depuis Descartes - ne sont évidemment pas pleinement développées : elles sont à peine esquissées. Il serait d'ailleurs déraisonnable, semble-t-il, selon les théoriciennes de l'écoféminisme, de s'attendre à un tel résultat à ce stade. Le développement de telles alternatives ne requiert pas seulement un effort de rationalisation fondamentale; il doit aussi, et peut-être prioritairement, émerger de pratiques alternatives, de modes de vie qui intègrent ce que la culture dualiste a séparé.

Ainsi, nous voyons que, pour cette position, toute tentative sérieuse de transfigurer la dichotomie femme/homme doit inclure une réarticulation $\mathrm{du}$ cadre conceptuel dualiste, rejaillissant ainsi sur d'autres parties clés des réseaux de dichotomies. Cette réarticulation doit englober les présupposés concernant la primauté de la sphère intellectuelle-rationnelle sur la sphère inférieure de la nature, et de la réalité humaine sur la réalité « naturelle » (dimension que l'on conçoit comme dénuée d'esprit), contrastes que des penseures telles que Simone de Beauvoir acceptent sans critique.

\section{Conclusion}

L'écoféminisme, courant de pensée multiple et en pleine formation, apparaît donc à qui se penche sur ses fondements comme une position encore relativement indistincte. Pourtant, au-delà des lacunes de ses perceptions politiques et épistémologiques, il semble fertile en promesses, ne serait-ce que par cette tentative, qu'il avoue lui-même indisciplinée et quelque peu chaotique, d'unir les intérêts de la libération des femmes, des institutions politiques nécessaires à la poursuite d'une vie sociale, et de la nature non humaine en une recherche globale de liberté, de paix, de créativité et de respect de l'harmonie naturelle. Nous avons à apprendre de la biosphère, suggèrent les écoféministes en reprenant ainsi un des credos du mouvement de « deep ecology », et nous nous devons de lui laisser l'espace d'une « parole » non humaine, verbe créateur s'il en fut. L'écoute et le respect de la nature ne signifient pas un retour à la vie des cavernes, mais bien plutôt la conscience de la richesse d'une évolution en parallèle, d'une façon complice, avec circonspection.

De plus, les femmes retrouvent, dans ce nouveau dialogue avec la nature non humaine la possibilité d'établir les bases compréhensives des mensonges idéologiques perpétrés par le patriarcat, en reprenant, depuis le début, le discours historique afin d'en extirper les derniers effets, les plus pervers, ceux qui se trouvent, encore aujourd'hui, fermement enracinés dans les processus de conceptualisation qui gèrent nos mondes. Il semble donc qu'aux yeux des écoféministes, renouer le dialogue entre femmes et nature sur une base engageant la redéfinition de la responsabilité, non seulement ne constitue en rien un recul par rapport aux avancées techno-scientifiques, mais devient le seul avenir possible d'une politique planétaire - raisonnable et sensible. 


\section{Références}

Beauvoir, Simone de, Le deuxième sexe, Paris, Gallimard, 1986 (ig49).

Firestone, Shulamith, La dialectique du sexe, Paris, Stock, 1972.

Jaggar, Alison M., Bordo, Susan R. (eds), Gender, Body, Knowledge : Feminist Reconstructions of Being and Knowing, New Brunswick and London, Rutgers University Press, I9go.

James, E. O., Le culte de la déesse-mère dans l'histoire des religions, Payot, Paris, 1960.

Jonas, Hans, The Imperative of Responsability : In Search of an Ethics for the Technological Age, Chicago, University of Chicago Press, 1984.

King, Ynestra, « Healing the Wounds : Feminism, Ecology, and Nature/Culture Dualism », in Jaggar/Bordo, I9go.

Ruddick, Sara, Maternal Thinking :Toward a Politics of Peace, Ballantine Books, New York, 1990. 